\title{
On Stable Calculation of Linear Functionals
}

\author{
By Sven-Åke Gustafson
}

\begin{abstract}
In this paper we discuss the recurrent task of evaluating a linear functional defined by (generally infinitely many) linear constraints. We develop a theory for the stability of this problem and suggest a regularization procedure, based on orthogonal expansions. Simple and efficient computational schemes for evaluating the functional numerically are given.
\end{abstract}

1. Calculation of Linear Functionals from Moment Conditions. We start by discussing the introductory

Example. $b \in C[0,1]$, i.e. $b$ is a continuous function over $[0,1]$. Introduce the maximum norm on $C[0,1]$. Let $L$ be a bounded linear functional. Consider the problem

$$
\text { Compute } L(b) \text { when }
$$

$$
L\left(a_{r}\right)=c_{r}, \quad r=1,2, \ldots,
$$

where $a_{r}(t)=t^{r-1}$.

As a particular instance of the problem (1) and (2) we take

$$
L(b)=\int_{0}^{1} b(t) \ln \left(t^{-1}\right) d t .
$$

Then

$$
c_{r}=L\left(a_{r}\right)=r^{-2}, \quad r=1,2, \ldots
$$

We shall call (2) moment conditions.

Lemma 1. Use the same notations and assumptions as in Example 1. Then $L(b)$ is uniquely determined by the sequence $c_{r}=L\left(a_{r}\right), r=1,2, \ldots$.

Proof. Let $b_{n}$ be the polynomial of degree less than $n$ which approximates $b$ best in the maximum norm. $b_{n}$ is uniquely determined and $\left\|b-b_{n}\right\| \rightarrow 0$ when $n$ $\rightarrow \infty$. Hence $L(b)=\lim _{n \rightarrow \infty} L\left(b_{n}\right)$, and the conclusion follows. Q.E.D.

However, by practical calculations $c_{r}$ are known only with a finite accuracy and only finitely $y_{i}$ many of the conditions (2) may be taken into account. Hence, a certain error is introduced in the calculated value of $L(b)$ which is determined by approximating $b$ (directly or indirectly) with linear combinations of $a_{1}, a_{2}, \ldots, a_{n}$. The purpose of this paper is to extend and generalize the results in [6] and [7] as well as to de-

Received March 23, 1978; revised July 14, 1978

AMS (MOS) subject classifications (1970). Primary 65J05, 65F25; Secondary 65B10, 65D30.

Key words and phrases. Linear functionals, moment condition, linear space, error bound, duality lemma. 
scribe efficient computational schemes for evaluating $L(b)$ and assessing the associated error.

Definition 1. Let $S$ be a fixed set, $F(S)$ the linear space of real-valued functions, defined on $S$. Thus, if $f, f_{1} \in S$ and $\alpha$ is a real number, we define as usual $f+f_{1}$ and $\alpha \cdot f$ through

$$
\left(f+f_{1}\right)(s)=f(s)+f_{1}(s) ; \quad(\alpha f)(s)=\alpha f(s), \quad s \in S .
$$

If $a_{r} \in F(s), r=1,2, \ldots, n$, we denote by $a$ the vector-valued function whose value $a(s), s \in S$, is given by the column vector with components $a_{r}(s), r=1,2, \ldots, n$. If $L$ is a linear functional defined on $F(S)$, we write $L(a)$ for the column vector with components $L\left(a_{r}\right), r=1,2, \ldots, n$. Thus, $L(a) \in R^{n}$, the $n$-dimensional Euclidean space.

Lemma 2. Let $a_{1}, a_{2}, \ldots, a_{n}, b$ and $L$ be as in Definition 1 and let $b$ be $a$ linear combination of $a_{1}, a_{2}, \ldots, a_{n}$,

$$
b(s)=y^{T} a(s), \quad s \in S,
$$

(where $y \in R^{n}$ and superscript ${ }^{T}$ as usual denotes transposition). Put

$$
L(a)=c
$$

Then

$$
L(b)=c^{T} y
$$

Proof.

$$
c^{T} y=y^{T} c=y^{T}(L a)=L\left(y^{T} a\right)=L(b) . \quad \text { Q.E.D. }
$$

Remark. For examples and computational applications of Lemma 2 see [6] and [8]. In the special case when $S$ is a finite set, $S=\left\{s_{1}, s_{2}, \ldots, s_{N}\right\}$, the functions $a_{r}$ may be represented as vectors in $R^{N}$. Since $L\left(a_{r}\right)$ then may be represented in the form of scalar products, (5) takes the form of linear systems of equations.

We next establish a more general result.

TheOREM 1. $S, a_{1}, a_{2}, \ldots, a_{n}$ and $L$ are as in Definition $1 . b \in F(S)$ and $y \in$ $R^{n}$. Introduce $\epsilon$ in $F(S)$ through

$$
y^{T} a=b+\epsilon .
$$

Let further $c, \delta$ in $R^{n}$ satisfy

$$
L(a)=c+\delta
$$

Then

$$
c^{T} y-L(b)=L(\epsilon)-\delta^{T} y
$$

Proof.

$$
c^{T} y-L(b)=y^{T} c-L\left(y^{T} a-\epsilon\right)=y^{T}(c-L(a))+L(\epsilon)=L(\epsilon)-\delta^{T} y . \quad \text { Q.E.D. }
$$

The rest of the paper will be based on Theorem 1. We demonstrate first how it can be used to derive bounds on $L(b)$, provided $\epsilon$ and $L$ meet certain further conditions. 
Definition 2. $L$ is called a nonnegative linear functional $(L \geqslant 0)$, if $f(s) \geqslant 0, s$ $\in S$, implies $L(f) \geqslant 0$.

COROLLARY 1. Use the same notations as in Theorem 1 and require further that $\delta=0$ and $L \geqslant 0$. Then

$$
y^{1 T} a(s) \leqslant b(s) \leqslant y^{2 T} a(s), \quad s \in S,
$$

implies

$$
y^{1 T} c \leqslant L(b) \leqslant y^{2 T} c
$$

Further put $v_{1}=\sup L(b)$ over all $L \geqslant 0$ such that $L(a)=c$ and $v_{2}=\inf c^{T} y$ over all $y \in R^{n}$ such that $y^{T} a(s) \geqslant b(s), s \in S$, then

$$
v_{1} \leqslant v_{2}
$$

Proof. With $\delta=0,(8)$ entails $L(b)=c^{T} y-L(\epsilon)$. Since $L \geqslant 0, L(b) \leqslant c^{T} y$, if $y^{T} a(s) \geqslant b(s), s \in S$, while $y^{T} a(s) \leqslant b(s)$ implies $L(b) \geqslant c^{T} y$. Thus (9) and (10) follow. Q.E.D.

Remark. (9) may be used to find bounds for $L(b)$. See [7] . (10) is a slight generalization of the duality lemma of semi-infinite programming, [4, Chapter II] and [10]. If $S$ is a finite set, then (10) coincides with the duality inequality of linear programming. The conditions of Corollary 1 can generally not be met in practice, since the exact values of $c_{r}$ cannot be represented in the computer. Thus, we arrive at the more general result.

Corollary 2. Use the same notations as in Theorem 1. Put $b_{n}=y^{T_{a}}$ and let the linear functional $L$ satisfy $L a=c$. Let further $u>0$ be a given (small) number and let $\bar{c}_{r}$ be such that

$$
\left|\bar{c}_{r}-c_{r}\right| \leqslant u\left|c_{r}\right|, \quad r=1,2, \ldots,
$$

then

$$
\left|L\left(b_{n}\right)-y^{T} \bar{c}\right| \leqslant u \sum_{r=1}^{n}\left|y_{r} c_{r}\right| \leqslant u \max _{1 \leqslant r \leqslant n}\left|c_{r}\right| \sum_{r=1}^{n}\left|y_{r}\right|
$$

Proof. Put $\epsilon=0$ and $\left|\delta_{r}\right| \leqslant u\left|c_{r}\right|$ in (8). Q.E.D.

Definition 3. Use the notations of Corollary 2 and put for $y^{T} \neq \neq 0$,

$$
\kappa_{n}=\sum_{r=1}^{n}\left|y_{r} c_{r}\right| /\left|y^{T} c\right|
$$

We shall call $\kappa_{n}$ the condition number of the problem to evaluate $L\left(b_{n}\right)$ from $L(a)$ $=c$.

Remark. $\kappa_{n} u$ is an upper bound for the relative error in the value of $L\left(b_{n}\right)$ caused by a relative error in the components of $c_{r}$, bounded by $u$. However, we shall be interested in comparing various sequences $\left\{b_{n}\right\}^{\infty}$, of approximations to a fixed $b$. When $L(b), L$ given is sought, then it turns out to be simpler to use 


$$
k_{n}=\max _{1 \leqslant r \leqslant n}\left|c_{r}\right| \sum_{r=1}^{n}\left|y_{r}\right|
$$

as a measure of stability. We have namely $\kappa_{n}\left|y^{T} c\right| \leqslant k_{n}$; and hence, $k_{n} u$ is a bound for the absolute error in $L\left(b_{n}\right)$ caused by a relative error in the components $c_{r}$, bounded by $u$. When we want to determine $L(b)$ for a general $b$, we first approximate $b$ by $b_{n}$. Combining (8) with Corollary 2 and setting $\epsilon=b_{n}-b$, we get

$$
\left|c^{T} y-L(b)\right| \leqslant\left|L\left(b_{n}-b\right)\right|+u \cdot k_{n} .
$$

If we select a sequence of functions $b_{n}, n=1,2, \ldots$, such that $L\left(b_{n}-b\right) \rightarrow 0$ when $n \rightarrow \infty$, we achieve that the first term of the right-hand side of (13) decreases with $n$. However, $k_{n}$ often increases with $n$ and hence there is an optimal value, $n=$ $n_{0}$, for which the right-hand side assumes its minimum value. $n_{0}$ depends on $b$, the approximating sequence $b_{1}, b_{2}, \ldots$ and $u$. We shall illustrate this fact on some simple but important problems in Section 2.

2. Stability of Some Convergence Acceleration Formulas. In this section we treat the problem: Let $L$ be a bounded linear functional on $C[0,1]$ when this space is equipped with the maximum norm. Put $c_{r}=L\left(a_{r}\right)$ for $a_{r}(s)=s^{r}, r=0,1, \ldots$. Compute for complex $z$

$$
F(z)=\sum_{r=0}^{\infty} c_{r}(-z)^{r}
$$

using $c_{0}, c_{1}, \ldots$ as input data. As explained in [9] this problem can be cast into the form: Compute

$$
F(z)=L(f(z, \cdot))
$$

for

$$
L\left(a_{r}\right)=c_{r}, \quad r=0,1, \ldots, \quad f(z, s)=(1+s z)^{-1} .
$$

Here (15) furnishes the analytic continuation of the function (14) to all $z$ outside the set defined by $z$ real and $z \leqslant-1$.

We investigate the stability of the convergence acceleration methods in Sections 2 and 3 of [9] and prove

THEOREM 2. Let $b_{n}$ be the polynomial of degree $n-1$ obtained by developing $(1+z s)^{-1}$ in a Taylor expansion around $s=t$ and retaining the first $n$ terms. Put $B=|z|(1+|t|) /|1+z t|$. Then the condition number $k_{n}$ of (13) has the properties $k_{n} / B^{n}$ is bounded for $n>1$, if $B>1$, $k_{n} / n$ is bounded for $n>1$, if $B=1$, $k_{n}$ is bounded for $n>1$, if $B<1$.

Proof.

$$
b_{n}(s)=\sum_{r=0}^{n-1} y_{r} r^{r}=(1+t z)^{-1} \sum_{r=0}^{n-1}\left\{\frac{z(t-s)}{1+t z}\right\}^{r}
$$


Thus

$$
\sum_{r=0}^{n-1}\left|y_{r}\right| \leqslant \frac{1}{|1+t z|} \sum_{r=0}^{n-1} B^{r}
$$

and hence,

$$
k_{n} \leqslant \frac{\max _{0 \leqslant r \leqslant n-1}\left|c_{r}\right|}{|1+t z|}\left(1+B+\cdots+B^{n-1}\right)
$$

establishing the desired result, since $c_{r}$ is bounded, $r \geqslant 1$. Q.E.D.

Remark. It may be of interest to compare the accuracy of the results reported in Table 2 of [9] with the estimates for $k_{n}$ obtainable from Theorem 2. Thus $t=1$, $z=1$ gives $B=1$, and by (17) $k_{n} \leqslant 0.5 . n \max _{0 \leqslant r \leqslant n-1}\left|c_{r}\right|$ in this case (Euler transformation), $t=1, z=10$ gives $B=20 / 11$ (generalized Euler transformation), and $t=$ $1 / 2, z=10$ gives $B=5 / 2$. The bounds for $k_{n}$ are sufficiently large to explain the observed loss in accuracy in our estimate of $F(z)$ for $z=10$. For each $n$ the bound for $k_{n}$ is somewhat smaller for $t=1$ than for $t=1 / 2$. However, for $t=1$ the optimal $n$-value is $n=30$ but for $t=1 / 2$ it is $n=20$ and the corresponding values of $k_{n}$ for optimal $n$ turn out to be of the same magnitude.

We next study the stability of the Čebyšev acceleration, which is described in [9, Section 3]. For this purpose we need

LemmA 3. Let the function $g$ satisfy $(-1)^{k} g^{(k)}(s)>0, k=0,1, \ldots$ for $s \in$ $[0,1]$ and let $Q(s)=\sum_{r=0}^{n-1} y_{r} s^{r}$, interpolate $g$ at $t_{1}, t_{2}, \ldots, t_{n}$ where $0 \leqslant t_{1}<t_{2}$ $<\cdots<t_{n} \leqslant 1$. Then

$$
\sum_{r=0}^{n-1}\left|y_{r}\right|=Q(-1)
$$

Further, if also $(-1)^{k} g^{(k)}(s)>0, k=0,1, \ldots, s \in[-1,1]$, then $Q(-1) \leqslant g(-1)$.

Remark. $g(s)=(1+s t)^{-1}, t>0$, and $g(s)=e^{-s}$ are examples of functions satisfying the assumptions in Lemma 3.

Proof. According to Newton's formula with divided differences we can write

$$
Q(s)=d_{0}+\left(s-t_{1}\right) d_{1}+\left(s-t_{1}\right)\left(s-t_{2}\right) d_{2}+\cdots+\left(s-t_{1}\right) \cdots\left(s-t_{n-1}\right) d_{n-1} \text {, }
$$

where $d_{0}=g\left(t_{1}\right), d_{1}=g\left(t_{1}, t_{2}\right), \ldots, d_{n-1}=g\left(t_{1}, t_{2}, \ldots, t_{n}\right)$. As known, there is a $\xi_{k} \in(0,1)$ such that $k ! d_{k}=g^{(k)}\left(\xi_{k}\right)$. Hence $(-1)^{k} d_{k}>0, k=0,1, \ldots, n-$ 1. Rewriting $Q$ in power form, we get an expression

$$
Q(s)=\sum_{r=0}^{n-1} y_{r} s^{r} \quad \text { with } y_{r}=(-1)^{r} h_{r}, h_{r}>0 .
$$

Thus

$$
Q(-1)=\sum_{r=0}^{n-1}\left|y_{r}\right|
$$

proving the first assertion. Newton's interpolation formula with remainder gives 


$$
g(-1)=Q(-1)+\frac{g^{(n)}(\xi)}{n !} \prod_{i=1}^{n}\left(-1-t_{i}\right),
$$

where $\xi \in[-1,1]$. Since $(-1)^{n} g^{(n)}(\xi)>0$ and $t_{i} \in[0,1]$, we get $Q(-1) \leqslant g(-1)$. Q.E.D.

Lemma 4. Put $g(t)=(|1+z|-|z|+t|z|)^{-1}, h(t)=(1+z t)^{-1}$ where $\operatorname{Re}(z)$ $>-1 / 2$, and let $0 \leqslant t_{1}<t_{2}<\cdots<t_{n} \leqslant 1$ be fixed points. Then the divided differences satisfy

$$
\left|h\left(t_{1}, t_{2}, \ldots, t_{k}\right)\right| \leqslant(-1)^{k-1} g\left(t_{1}, t_{2}, \ldots, t_{k}\right), \quad k=1,2, \ldots, n .
$$

Further if

$$
Q(s)=\sum_{r=0}^{n-1} y_{r} s^{r}, \quad R(s)=\sum_{r=0}^{n-1} w_{r} s^{r}
$$

interpolate $g$ and $h$ respectively at $t_{1}, t_{2}, \ldots, t_{n}$, then

$$
\sum_{r=0}^{n-1}\left|w_{r}\right| \leqslant \sum_{r=0}^{n-1}\left|y_{r}\right|
$$

Proof. We have

$$
\begin{gathered}
h\left(t_{1}, t_{2}, \ldots, t_{k}\right)=(-z)^{k-1} \prod_{i=1}^{k}\left(1+z t_{i}\right)^{-1} \\
g\left(t_{1}, t_{2}, \ldots, t_{k}\right)=(-1)^{k-1}|z|^{k-1} \prod_{i=1}^{k}\left(|1+z|-|z|+t_{i}|z|\right)^{-1}
\end{gathered}
$$

For $\operatorname{Re}(z)>-1 / 2,|1+z|>|z|$. Hence by $(20),(-1)^{k-1} g\left(t_{1}, t_{2}, \ldots, t_{k}\right)$ $>0$. Since $|z|\left(|1+z|-|z|+t_{i}|z|\right)^{-1} \geqslant\left|z\left(1+z t_{i}\right)^{-1}\right|,(18)$ follows. In order to verify (19) we express $Q$ and $R$ by means of Newton's formula with divided differences and evaluate $y_{r}$ and $w_{r}$. Then the conclusion follows as in Lemma 3. Q.E.D.

We may now prove

THEOREM 3. Let $Q_{n}=\sum_{r=0}^{n-1} y_{r} t^{r}$ be the polynomial of degree less than $n$ which interpolates $(1+z t)^{-1}$ at the zeros of $T_{n}^{*}$ where $T_{n}^{*}$ is the shifted $\breve{C}$ ebyšev polynomial of degree $n$ defined by $T_{n}^{*}(x)=T_{n}(2 x-1)$. Here $T_{n}$ is the usual Čebyšev polynomial of degree $n$. See [2]. Then

$$
\begin{gathered}
\sum_{r=1}^{n}\left|y_{r}\right| \leqslant(1-z)^{-1}, \quad \text { if } z \text { real and } z \in(0,1), \\
\sum_{r=1}^{n}\left|y_{r}\right| \leqslant(|1+z|-2|z|)^{-1}, \quad \text { if }|z-1 / 3|<2 / 3 .
\end{gathered}
$$

Thus, if $z$ meets the requirements indicated in (21) or (22), then the condition number $k_{n}$ of the Čebyšev-acceleration in Section 3 of [9] remains bounded for all $n$.

Proof. If $z \in[0,1]$, then $(1+z t)^{-1}$ meets the assumptions of Lemma 3 and (21) holds. (22) is established by Lemma 4, since if $|z-1 / 3|<2 / 3$, then $\operatorname{Re}(z)>$ $-1 / 3$. Thus, we may replace $(1+z t)^{-1}$ by $(|1+z|-|z|+t|z|)^{-1}$ and apply Lemma 3 to that function, and the conclusion follows. Q.E.D. 
Remark. If $z$ real and $z>1$, we may still apply Lemma 3 to find $\sum_{r=1}^{n}\left|y_{r}\right|=$ $Q_{n}(-1)$, where $Q_{n}$ is defined as in Theorem 3. Using the recurrence relation for $T_{n}^{*}$ we establish as in Section 3 of [9] that $Q_{n}(-1)$ increases exponentially with $n$. If $z$ is complex but does not meet the condition associated with (22), we get the inequality

$$
\sum_{r=1}^{n}\left|y_{r}\right| \geqslant\left|Q_{n}(-1)\right|
$$

which gives lower bounds for the condition number. These are exponentially growing with $n$, if $\max \left(\lambda_{1}, \lambda_{2}\right)>1$ where $\lambda_{1}=\left|2 z^{-1}+1+2 \sqrt{z^{-2}+z^{-1}}\right|, \lambda_{2}=1 / \lambda_{1}$. Compare [9, Section 3]. Thus Theorems 2 and 3 are consistent with the numerical results reported in [9] which indicated the existence of an optimal $\boldsymbol{n}$ of modest magnitude giving the best estimate of $L(b)$ in (13).

\section{Calculation of Linear Functionals by Means of Orthogonal Expansions.}

Definition 4. Let $F(S)$ be as in Definition 1 and let $d \alpha$ be a nonnegative measure over $S$. We denote by $L_{\alpha}^{2}(S)$ the space of functions which are square-integrable over $S$ with respect to $d \alpha$ and equipped with the scalar product

$$
\langle f, g\rangle=\int_{S} f(s) g(s) d \alpha(s)
$$

for $f, g$ in $L_{\alpha}^{2}(S)$ and the norm given by $\|f\|_{2}^{2}=\langle f, f\rangle$. The functions $a_{1}, a_{2}, \ldots, a_{n}$ are said to be linearly independent in $L_{\alpha}^{2}(S)$, if $\left\|\sum_{r=1}^{n} z_{r} a_{r}\right\|_{2}=0$ implies $z_{1}=z_{2}=$ $\cdots=z_{n}=0$.

We prove

LEMMA 5. Let $a_{1}, a_{2}, \ldots, a_{n}$ be an orthonormal system in $L_{\alpha}^{2}(S)$ and put

$$
b_{n}=y^{T} a \text { with } y_{r}=\left\langle b_{n}, a_{r}\right\rangle
$$

Then

$$
\sum_{r=1}^{n}\left|y_{r}\right| \leqslant \sqrt{n}\left\|b_{n}\right\|_{2}
$$

Proof. Since $a_{1}, a_{2}, \ldots, a_{n}$ are orthonormal, $y^{T} y=\left\|b_{n}\right\|_{2}^{2}$. Next maximize $\sum_{r=1}^{n}\left|y_{r}\right|$ under the condition $y^{T} y=\left\|b_{n}\right\|_{2}^{2}$ and (24) follows. Q.E.D.

Thus, $k_{n}$ increases at most as $\sqrt{n}$, if $a_{1}, a_{2}, \ldots, a_{n}$ meet the conditions of Lemma 5. Consider again the problem:

Estimate $L(b)$ when $L\left(a_{r}\right)=c_{r}, r=1,2, \ldots, n$, are given numerically and $a_{1}$, $a_{2}, \ldots, a_{n}$ are linearly independent in the sense of Definition 4 for a certain measure $d \alpha$. In view of Lemma 5 it may be advantageous first to orthogonalize $a_{1}, a_{2}$, $\ldots, a_{n}$ in a preliminary step and then approximate $L(b)$ with $L\left(b_{n}\right)$. This type of stabilization of a problem is often advantageous. See [3, Chapter 1]. If this orthogonalization must be carried out numerically for a general system, then the modified Gram-Schmidt method [1] should be used in order to secure numerical stability of the transformation.

We describe now how to perform the transformation in the important case when $S$ is a real interval and $a_{r}(s)=s^{r-1}$. Then we select $d \alpha$ such that the corresponding system $q_{0}, q_{1}, \ldots$, of orthogonal polynomials has a three-term recurrence relation 
with coefficients $u_{r}, v_{r}$, which are known as analytic expressions,

$$
\begin{gathered}
q_{0}(s)=1, \quad q_{1}(s)=s-u_{1}, \\
q_{n}(s)=\left(s-u_{n}\right) q_{n-1}(s)-v_{n} q_{n-2}(s), \quad n=2,3, \ldots .
\end{gathered}
$$

(The so-called classical orthogonal families: Jacobi, Hermite, Laguerre, etc., meet these requirements.) Afterwards, we normalize $q_{0}, q_{1}, \ldots$ by putting

$$
e_{r}=w_{r} q_{r-1}
$$

where the constant $w_{r}$ is determined (analytically) to render $\left\|e_{r}\right\|=1$. There is a discrete measure $d \alpha_{n}$,

$$
\int_{S} f(s) d \alpha_{n}(s)=\sum_{i=1}^{n} m_{n i} f\left(s_{n i}\right)
$$

such that $m_{\text {in }}>0, i=1,2, \ldots$, and

$$
\int_{s} e_{j}(s) e_{k}(s) d \alpha_{n}(s)=\delta_{j k}, \quad 1 \leqslant j \leqslant k<n .
$$

$m_{n i}$ and $s_{n i}$ are weights and abscissae in the $n$-point Gaussian quadrature rule corresponding to the measure $d \alpha$ and are efficiently calculated using the computer codes in [5]. (29) follows from the relation

$$
\delta_{j k}=\int_{S} e_{j}(s) e_{k}(s) d \alpha(s)=\sum_{i=1}^{n} m_{n i} e_{j}\left(s_{n i}\right) e_{k}\left(s_{n i}\right)=\int_{s} e_{j}(s) e_{k}(s) d \alpha_{n}(s),
$$

which is valid since the $n$-point Gaussian rule gives an exact result for polynomials of degree $2 n-1$ or less.

We next determine $L q_{r}$ which is done by means of the recurrence relations (25), (26). Put $f_{k r}(s)=s^{k} q_{r}(s) l_{k r}=L\left(f_{k r}\right)$. We first calculate the auxiliary entities $l_{k r}$ for $k+r<n, k=0,1, \ldots, n-1$. (25), (26) give the relationships

$$
\begin{gathered}
l_{k, 0}=c_{k+1}, \quad k=0,1, \ldots, \\
l_{k, 1}=c_{k+2}-u_{1} c_{k+1}, \quad k=0,1, \ldots, \\
l_{k, n}=l_{k+1, n-1}-u_{n} l_{k, n-1}-v_{n} l_{k, n-2}, \quad n=2,3, \ldots .
\end{gathered}
$$

We arrange the numbers $l_{k, n}$ in a triangular array

$$
l_{0,0}
$$

$$
\begin{array}{ccc} 
& l_{0,1} & \\
l_{1,0} & & l_{0,2} \\
& l_{1,1} & \\
l_{2,0} & & l_{1,2} \\
& & \vdots \\
& l_{2,1} & \vdots \\
l_{3,0} & \vdots &
\end{array}
$$


By (27), $L\left(e_{r}\right)=w_{r} L\left(q_{r-1}\right)=w_{r} l_{0, r-1}, r=1,2, \ldots, n$. The desired elements $l_{0, r}$ appear in the top of the columns of the array (33). The numbers $l_{k r}$ are calculated along ascending diagonals, i.e. in the order $l_{0,0}, l_{1,0}, l_{0,1}, l_{2,0}, l_{1,1}, l_{0,2}, \ldots$ Then, it follows from (30), (31), (32) that in order to calculate the elements in a certain ascending diagonal one needs access only to the two preceding diagonals. Hence, it is not necessary to store the entire array (33) simultaneously. In total about $n^{2}$ addition/subtractions and about $n^{2}$ multiplications are required for determining $L(e)$.

Next we put $b_{n}=y^{T} e$, where $y$ is the optimal solution of

$$
\min _{z \in R^{n}} \int_{S}\left[b(s)-z^{T} e(s)\right]^{2} d \alpha_{n}(s) .
$$

If we take $z^{T} e$ as the unique polynomial of degree $n$ which interpolates $b$ at the zeros $s_{n i}$ of $e_{n}$, then by (28) the integral (34) assumes the value 0 ; and hence, this $z^{T} e$ is optimal. But as known, the unique optimal solution of the least-squares problem (34) is given by $z=y$, where

$$
y_{r}=\int_{S} b(s) e_{r}(s) d \alpha_{n}(s)=\sum_{i=1}^{n} m_{n i} b\left(s_{n i}\right) e_{r}\left(s_{n i}\right) .
$$

The calculation of $y$ requires about $n^{2}$ multiplications and the same number of additions. Now to determine $L\left(b_{n}\right)$ only the computation of a further scalar product is called for.

Remark. If $n$ is increased, $d \alpha_{n}$ is changed and most of the work including calculating functional values $b\left(s_{n i}\right)$ must be redone from scratch.

We next discuss the case when $S$ is a bounded interval, and we shall assume that by a linear transformation it has been transformed into the standard interval $[-1,1]$. Then it is often suggested to take $b_{n}$ as the polynomial of degree $<n$ which interpolates $b$ at the zeros of the Čebyšev polynomial of degree $n, T_{n}(x)$. See, e.g., $[3],[12]$ and [14]. Then

$$
s_{n i}=\cos \frac{i-1 / 2}{n} \pi, \quad m_{n i}=\pi / n .
$$

If the cost to evaluate $b\left(s_{i n}\right)$ is great in comparison to an arithmetic operation and it is not known which $n$-value is finally accepted, then one wants to avoid discarding previously calculated functional values. In this situation [11] suggests that one should start with an odd $n$-value, advance $n$ according to

$$
n_{\text {new }}=2 \cdot n_{\text {old }}+1
$$

and select $s_{n i}$ as the zeros of $U_{n}$, the $n$th degree Čebyšev polynomial of the second kind. Thus,

$$
s_{n i}=\cos \frac{\pi i}{n+1}, \quad m_{n i}=\frac{1}{n+1} \sin ^{2} \frac{\pi i}{n+1} .
$$

From (37) we find that when $n$ is advanced according to (36), all earlier computed functional values are retained. 
It is known, that if $b$ may be continued analytically to an ellipse in the complex plane with foci at +1 and $-1, s_{n i}$ are given by (35) and $b_{n}$ is the polynomial of degree $<n$ which interpolates $b$ at $s_{n i}$, then

$$
\left|b(s)-b_{n}(s)\right| \leqslant A \lambda^{n}
$$

where $A$ and $\lambda$ are constants and $\lambda<1$. But if $s_{n i}$ are given by (36), then we get a bound

$$
\left|b(s)-b_{n}(s)\right| \leqslant n A_{0} \lambda^{n},
$$

where $A_{0}$ is another constant and $\lambda$ has the same value as in (37). See [11]. A special case occurs in the Čebyšev acceleration scheme treated in Section 2 and in [9]. The bounds (38) and (39) are easily established by means of a straightforward application of Cauchy's integral formula.

Let $\Delta$ be a bound for the magnitude of the absolute error in the value of $c_{r}$. If $q_{n}(t)=z^{T} a(t)$ for some $z \in R^{n}$, then the resulting error in $L\left(q_{n}\right)$ is bounded by $\Delta \sum_{r=1}^{n}\left|z_{r}\right|$. We may derive simple expressions for this bound for $q_{n}=T_{n}$ and $q_{n}$ $=U_{n}$ by means of:

LEMMA 6. Let $t_{n}$ and $u_{n}$ denote the sums of the absolute values of the coefficients of $T_{n}$ and $U_{n}$, the nth degree Čebyšev polynomials of first and second kind. Then

$$
\begin{aligned}
& t_{n}=1 / 2\left[(1+\sqrt{2})^{n}+(1-\sqrt{2})^{n}\right] \\
& u_{n}=\frac{1}{2 \sqrt{2}}\left[(1+\sqrt{2})^{n+1}-(1-\sqrt{2})^{n+1}\right] .
\end{aligned}
$$

Proof. It is well known that

$$
\begin{aligned}
& T_{n}(z)=1 / 2\left\{\left(z+\sqrt{z^{2}-1}\right)^{n}+\left(z-\sqrt{z^{2}-1}\right)^{n}\right\}, \\
& U_{n}(z)=\frac{1}{2 \sqrt{z^{2}-1}}\left\{\left(z+\sqrt{z^{2}-1}\right)^{n+1}-\left(z-\sqrt{z^{2}-1}\right)^{n+1}\right\} .
\end{aligned}
$$

Since $t_{n}=\left|T_{n}(i)\right|, u_{n}=\left|U_{n}(i)\right|$ the stated result follows immediately. Q.E.D.

Remark. The result for $t_{n}$ is also given in [13, p. 792, Eq. (14)]. We treat also the case $S=[0,1]$. Then we use the shifted Cebyšev polynomials $T_{n}^{*}$ and $U_{n}^{*}$ defined by $T_{n}^{*}(x)=T_{n}(2 x-1)$ and $U_{n}^{*}(x)=U_{n}(2 x-1)$. See e.g. [9]. Lemma 6 is replaced by

LEMMA 7. Let $t_{n}^{*}$ and $u_{n}^{*}$ denote the sums of the absolute values of the coefficients of $T_{n}^{*}$ and $U_{n}^{*}$. Then

$$
\begin{aligned}
& t_{n}^{*}=1 / 2\left[(1+\sqrt{2})^{2 n}+(1-\sqrt{2})^{2 n}\right] \\
& u_{n}^{*}=\frac{1}{4 \sqrt{2}}\left\{(1+\sqrt{2})^{2 n+2}+(1-\sqrt{2})^{2 n+2}\right\} .
\end{aligned}
$$
Q.E.D.

Proof. Use the fact that $T_{n}^{*}\left(x^{2}\right)=T_{2 n}(x)$ and $2 \times U_{n}^{*}\left(x^{2}\right)=U_{2 n+1}(x)$. 
Remark. It is apparent from the above that if $b_{n}=\sum_{r=1}^{n} y_{r} T_{r-1}$, the error in $L\left(b_{n}\right)$ computed with $L(a), a_{r}(s)=s^{r-1}$ as input data depends on how rapidly $y_{r}$ decreases with $r$. We have namely

$$
L\left(b_{n}\right)=\sum_{r=1}^{n} y_{r} L\left(T_{r-1}\right),
$$

and the errors in the calculated values of $L\left(T_{r-1}\right)$ are bounded by a quantity of the form $A(1+\sqrt{2})^{r}$ where $A$ is a constant.

Also, if the relative error in the calculated value of $L\left(T_{r}\right)$ is greater than $100 \%$ we might as well put $L\left(T_{r}\right)=0$ since this value is consistent with given data and associated error bounds. In this situation, the addition of more moments $c_{r}$ does not improve upon our estimate of $L(b)$.

Computing Research Group

The Australian National University

P. O. Box 4

Canberra A.C.T. 2600, Australia

1. Å. BJÖRCK, "Solving linear least squares problems by Gram-Schmidt orthogonalization," $B I T$, v. 7, 1967, pp. 1-21.

2. C. W. CLENSHAW, "Chebyshev series for mathematical functions," Mathematical Tables, v. 5, National Physical Laboratory, HMSO, London, 1962.

3. G. DAHLQUIST, A. BJÖRCK \& N. ANDERSON, Numerical Methods, Prentice-Hall, Englewood Cliffs, N. J., 1974.

4. K. GLASHOFF \& S.- $\AA$. GUSTAFSON, Einführung in die lineare Optimierung, Wissenschaftliche Buchgesellschaft, Darmstadt, 1978.

5. G. H. GOLUB \& J. H. WELSCH, "Calculation of Gauss quadrature rules," Math. Comp., v. 23, 1969 , pp. 221-230.

6. S.-A. GUSTAFSON, "Control and estimation of computational errors in the evaluation of interpolation formulas and quadrature rules," Math. Comp., v. 24, 1970, pp. 847-854.

7. S.-A. GUSTAFSON, "On computational applications of the theory of moment problems," Rocky Mountain J. Math., v. 4, 1974, pp. 227-240.

8. S.-A. GUSTAFSON, "Some optimization problems in numerical analysis," Methods of Operations Research, v. 25, 1977 , pp. 367-379.

9. S.- $\AA$. GUSTAFSON, "Convergence acceleration on a general class of power series," Computing, v. 21, 1978, pp. 367-379.

10. S.-A. GUSTAFSON \& K. O. KORTANEK, "Numerical treatment of a class of semiinfinite programming problems," Naval Res. Logist. Quart., v. 20,1973, pp. 477-504.

11. S.-A. GUSTAFSON \& S. LINDAHL, "Numerical computation of an integral appearing in the Fröman-Fröman phase-integral formula for calculation of quantal matrix elements without the use of wave functions," J. Computational Phys., v. 24, 1977, pp. 81-95.

12. I. MELINDER, "Accurate approximation in weighted maximum norm by interpolation," J. Approximation Theory, v. 22, 1978, pp. 33-45.

13. A.C. R. NEWBERY, "Error analysis for polynomial evaluation," Math. Comp., v. 28, 1974, pp. 789-793.

14. M. J. D. POWELL, "On the maximum norm of polynomial approximation defined by interpolation and by least squares criteria," Comput. J., v. 9, 1967, pp. 404-407.

15. T. RIVLIN, The Chebyshev polynomials, Wiley, New York, 1974. 